\title{
Gasdermins pore cell membrane to pyroptosis
}

\author{
Huanhuan Liang \& Yingfang Liu* \\ State Key Laboratory on Biomacromolecules, Institute of Biophysics, Chinese Academy of Sciences, Beijing 100101
}

Received August 8, 2016; accepted August 10, 2016; published online September 6, 2016

Citation: Liang, H., and Liu, Y. (2016). Gasdermins pore cell membrane to pyroptosis. Sci China Life Sci 59, 1090-1092. doi: 10.1007/s11427-016-0243-4

Cell death programmes including apoptosis, pyroptosis, autophagy, oncosis and necroptosis were defined dependent on distinct biochemical mechanism and genetic pathways. Pyroptosis is an inflammatory form of programmed cell death characterized by the activation of inflammatory caspases, cell swelling, pore formation on plasma membrane and rapid cellular rupture which cause the release of cell contents followed by robust inflammatory responses (Cookson and Brennan, 2001; Jorgensen and Miao, 2015). Two pathways have been uncovered for the activation of the inflammatory caspases. For one of the two pathways which is recognized as canonical pathway, in response to invading pathogens and endogenous danger signals, a type of multimeric protein complexes known as inflammasome is assembled, comprising sensor proteins (NLRs, ALRs, or pyrin), adaptor molecules usually using ASC, and zymogen pro-caspase-1 (Sharma and Kanneganti, 2016). The inflammasome provides a platform for the activation of caspase-1, which is responsible for the maturation and secretion of pro-inflammatory cytokines, interleukin (IL)-1 $\beta$ and IL-18 (Keller et al., 2008). Different from caspase-1, caspases-11 in mice and its ortholog caspase-4/5 in human are directly activated by cytosolic lipopolysaccharides (LPS) (Shi et al., 2014), this is termed as non-canonical pathway. It was reported that pores with diameter of 1.1-2.4 $\mathrm{nm}$ are produced on plasma membrane, dependent on the activation of caspase-1 during salmonella infection (Fink and Cookson, 2006). The pores were proposed to dissipate cellular ionic gradients, resulting in increased water influx and eventually membrane rupture. However, how these

*Corresponding author (email: liuyingfang@ibp.ac.cn) plasma-membrane pores are formed and regulated by inflammatory caspases had been unclear for a decade. Thus, to understand its detailed mechanisms, immunologists studied the substrates of activated inflammatory caspases and their downstream signaling pathways. Some significant breakthroughs have been made recently.

A crucial substrate of inflammatory caspases, gasdermin D (GSDMD), was independently identified by several groups in 2015 (He et al., 2015; Kayagaki et al., 2015; Shi et al., 2015). According to their results, GSDMD is cleaved by the activated caspases-1/4/5/11 between its $\mathrm{N}$-terminal and C-terminal domains. The $\mathrm{N}$ terminal domain of GSDMD (GSDMD-N) possesses intrinsic pyroptosis-inducing activity and determines the secretion of IL-1 $\beta$ and IL-18. Furthermore, most recently, the mechanism for the cytotoxicity of GSDMD-N in pyroptosis was dissected by four groups (Aglietti et al., 2016; Ding et al., 2016; Liu et al., 2016; Sborgi et al., 2016). The researchers expressed GSDMD-N or GSDMD with caspases-11 in human 293T cells, and found that cells developed swelling bubbles and became ruptured soon after GSDMD-N was produced. Therefore, they hypothesized that GSDMD-N might kill cells by disrupting the integrity of cell membrane, similar to mixed lineage kinase domain-like protein (MLKL) which oligomerized and associated to the inner leaflet of the plasma membrane to permeabilize the membrane during necroptotic cell death (Cai et al., 2014). Except for GSDMD, Ding et al found that the $\mathrm{N}$ terminal domain of most of gasdermin proteins (gasdermin-N) is highly toxic to 293T cells and Escherichia coli. Thus, they suggested gasdermin proteins, including but not limited to GSDMD, as general pyroptosis factors (Ding et al., 2016).

To test whether GSDMD-N directly or indirectly initiate 
a downstream event to disrupt the plasma membrane, the researchers studied the subcellular localization of GSDMD-N. Their results showed that full-length GSDMD localizes in the cytosol. However, GSDMD-N moves to plasma membrane and mitochondria quickly during pyroptosis. The membrane targeting processes of GFP labeled GSDMD-N were visualized using immunofluorescence microscopy. They further tested the binding ability of GSDMD-N to numerous lipids dotted on membrane or contained in liposome. The results showed that GSDMD-N specifically binds to constituents on the inner side of eukaryotic cell membranes, including phosphatidylinositol phosphates (PIPs), phosphatidic acid (PA) and phosphatidylserine (PS). In addition, it can also bind to cardiolipin, an important component of the bacterial as well as eukaryotic mitochondrial inner membranes. Similar results were also obtained using the $\mathrm{N}$ domains of GSDMA and GSDMA3. The lipid-binding preference of gasdermin proteins correlates well with the fact that gasdermins disrupt plasma membrane from inner leaflet of the cells, but do not harm living cells when administered extracellularly.

Although the full length GSDMD exists as monomer in solution, GSDMD-N oligomerizes in liposome and forms ring-like structure detected by cross-linking assay, native gel electrophoresis and negative stain electron microscopy. The multimer disassembles under reducing conditions, indicating that disulfide bonds are formed among GSDMD-N molecules. Taken together, these properties of GSDMD-N suggest they may oligomerize and form pores within plasma membrane. The researchers employed liposome-leakage experiment to verify this proposition. GSDMD-N and other gasdermin-Ns can disrupt phosphoinositide or cardiolipin-containing liposome, release $\mathrm{Tb}^{3+}, \mathrm{Ca}^{2+}$, polyethylene glycols (PEGs) or dextrans with increasing molecular weight from preloaded liposomes. To estimate the size of the GSDMD-dependent plasma membrane pore, the researchers employed electron microscopy and atomic force microscopy (AFM) to directly observe the pores formed by GSDMD-N on liposomes. The diameters of these pores were measured at approx. 10-20 nm, theoretically large enough for secretion of the proinflammatory cytokines interleukin (IL)-1 $\beta$ and IL-18. A 16-fold symmetry of GSDMA3 pores was also observed due to optimized protein/background contrast (Ding et al., 2016).

The pore formation activity of Gasdermin- $\mathrm{N}$ is triggered by inflammatory caspases cleavage, indicating an autoinhibition mechanism may exist for gasdermin proteins. To uncover its structure basis, Ding et al determined the autoinhibited conformation structure of full length GSDMA3 at $1.9 \AA$ resolution. The GSDMA3-N folds into a novel fold, which represents a new type of pore-forming protein structure. Using the $\mathrm{N}$ terminal 60 residues, GSDMA3-N closely interacts with globular folded GSDMA3-C domain. Mutations on the interface exhibited spontaneous pyroptosis-inducing activity. Based on homol- ogy based structure modeling and mutagenesis results, they suggested that the autoinhibition mechanism would be conserved in the gasdermin family.

These results revealed that gasdermin family proteins are direct and final executors of pyroptotic cell death, which closed the long-standing gap between inflammatory caspases activation and pyroptotic cell lysis. Meanwhile, new questions about the functions of gasdermin pores are raised. For instance, since gasdermin proteins can bind various phosphoinositides and associate with heterogeneous membranes during pyroptosis, it is possible that gasdermin- $\mathrm{N}$ may form pores on mitochondria, endosome and phagosome membranes, which are related with other cellular physiologies. Besides, gasdermin-N binds cardiolipin and kills bacteria in vitro, indicating that they may serve as anti-bacteria proteins by directly killing intracellular or extracellular bacteria during pyroptosis (Ding et al., 2016; Liu et al., 2016). Furthermore, although residues participating in oligomerization and membrane insertion were proposed, the structural basis of gasdermin-N pore formation remains to be fully elucidated. In addition to gasdermin, a cytotoxicity mediated by pannexin1-P2X7 was reported recently (Yang et al., 2015). Caspase-11-dependent cleavage of the pannexin-1 allows the release of ATP which further activates P2X7 to mediate pyroptosis. The correlation of these two pathways is unclear. Finally, since pyroptosis is associated with autoinflammatory conditions (Jorgensen and Miao, 2015), septic shock (Kayagaki et al., 2013), and HIV infection-CD4 T-cell depletion (Doitsh et al., 2014), functions of gasdermin proteins in these conditions need to be further studied for potential therapeutic application development.

Compliance and ethics The author(s) declare that they have no conflict of interest.

Aglietti, R.A., Estevez, A., Gupta, A., Ramirez, M.G., Liu, P.S., Kayagaki, N., Ciferri, C., Dixit, V.M., and Dueber, E.C. (2016). GsdmD p30 elicited by caspase-11 during pyroptosis forms pores in membranes. Proc Natl Acad Sci USA 113, 7858-7863.

Cai, Z., Jitkaew, S., Zhao, J., Chiang, H.C., Choksi, S., Liu, J., Ward, Y., Wu, L.G., and Liu, Z.G. (2014). Plasma membrane translocation of trimerized MLKL protein is required for TNF-induced necroptosis. Nat Cell Biol 16, 55-65.

Cookson, B.T., and Brennan, M.A. (2001). Pro-inflammatory programmed cell death. Trends Microbiol 9, 113-114.

Ding, J., Wang, K., Liu, W., She, Y., Sun, Q., Shi, J., Sun, H., Wang, D.C., and Shao, F. (2016). Pore-forming activity and structural autoinhibition of the gasdermin family. Nature 535, 111-116.

Doitsh, G., Galloway, N.L., Geng, X., Yang, Z., Monroe, K.M., Zepeda, O., Hunt, P.W., Hatano, H., Sowinski, S., Muñoz-Arias, I., and Greene, W.C. (2014). Cell death by pyroptosis drives CD4 T-cell depletion in HIV-1 infection. Nature 505, 509-514.

Fink, S.L., and Cookson, B.T. (2006). Caspase-1-dependent pore formation during pyroptosis leads to osmotic lysis of infected host macrophages. Cell Microbiol 8, 1812-1825.

He, W.T., Wan, H., Hu, L., Chen, P., Wang, X., Huang, Z., Yang, Z.H., Zhong, C.Q., and Han, J. (2015). Gasdermin D is an executor of pyroptosis and required for interleukin-1beta secretion. Cell Res 25, 1285-1298. 
Jorgensen, I., and Miao, E.A. (2015). Pyroptotic cell death defends against intracellular pathogens. Immunol Rev 265, 130-142.

Kayagaki, N., Stowe, I.B., Lee, B.L., O'Rourke, K., Anderson, K., Warming, S., Cuellar, T., Haley, B., Roose-Girma, M., Phung, Q.T., Liu, P.S., Lill, J.R., Li, H., Wu, J., Kummerfeld, S., Zhang, J., Lee, W.P., Snipas, S.J., Salvesen, G.S., Morris, L.X., Fitzgerald, L., Zhang, Y., Bertram, E.M., Goodnow, C.C., and Dixit, V.M. (2015). Caspase-11 cleaves gasdermin D for non-canonical inflammasome signalling. Nature 526, 666-671.

Kayagaki, N., Wong, M.T., Stowe, I.B., Ramani, S.R., Gonzalez, L.C., Akashi-Takamura, S., Miyake, K., Zhang, J., Lee, W.P., Muszyński, A., Forsberg, L.S., Carlson, R.W., and Dixit, V.M. (2013). Noncanonical inflammasome activation by intracellular LPS independent of TLR4. Science 341, 1246-1249.

Keller, M., Ruegg, A., Werner, S., and Beer, H.D. (2008). Active caspase-1 is a regulator of unconventional protein secretion. Cell 132, 818-831.

Liu, X., Zhang, Z., Ruan, J., Pan, Y., Magupalli, V.G., Wu, H., and Lieberman, J. (2016). Inflammasome-activated gasdermin D causes pyroptosis by forming membrane pores. Nature 535, 153-158.

Sborgi, L., Ruhl, S., Mulvihill, E., Pipercevic, J., Heilig, R., Stahlberg, H., Farady, C.J., Muller, D.J., Broz, P., and Hiller, S. (2016). GSDMD membrane pore formation constitutes the mechanism of pyroptotic cell death. EMBO J 35, 1766-1778.

Sharma, D., and Kanneganti, T.D. (2016). The cell biology of inflammasomes: Mechanisms of inflammasome activation and regulation. J Cell Biol 213, 617-629.

Shi, J., Zhao, Y., Wang, Y., Gao, W., Ding, J., Li, P., Hu, L., and Shao, F. (2014). Inflammatory caspases are innate immune receptors for intracellular LPS. Nature 514, 187-192.

Shi, J., Zhao, Y., Wang, K., Shi, X., Wang, Y., Huang, H., Zhuang, Y., Cai, T., Wang, F., and Shao, F. (2015). Cleavage of GSDMD by inflammatory caspases determines pyroptotic cell death. Nature 526, 660-665.

Yang, D., He, Y., Munoz-Planillo, R., Liu, Q., and Nunez, G. (2015). Caspase-11 requires the Pannexin-1 channel and the purinergic P2X7 pore to mediate pyroptosis and endotoxic shock. Immunity 43, 923-932.

Open Access This article is distributed under the terms of the Creative Commons Attribution License which permits any use, distribution, and reproduction in any medium, provided the original author(s) and source are credited. 